\title{
Late outcome after surgical and topical treatment for Aspergillus mediastinitis after heart transplantation
}

\author{
Paul Vogt ${ }^{1}$, Raed Aser ${ }^{2}$, Daniel Schmidlin ${ }^{1}$, Paul Mohacsi ${ }^{1}$, Pascal Berdat ${ }^{1}$, Philip Went ${ }^{3}$, \\ Markus Flepp ${ }^{1}$, and Carlos Mestres ${ }^{4}$ \\ ${ }^{1}$ Hirslanden Klinik im Park \\ ${ }^{2}$ UniversitätsSpital Zürich \\ ${ }^{3}$ Kantonsspital Graubünden \\ ${ }^{4}$ University Hospital Zurich
}

October 29, 2020

\begin{abstract}
Here we report the seven-year long-term survival after Aspergillus fumigatus mediastinitis after heart transplantation, an uncommonly described condition. A 66-year-old male developed infection with A. fumigatus covering the entire thoracic cavity with a fungal turf after orthotopic heart transplantation. Repeated surgical removal of infectious and necrotic tissue together with innovative topical treatment using voriconazole and chlorhexidine combined with systemic antifungal treatment lead to control of infection. Definitive wound closure was achieved by standard sternal refixation and latissimus dorsi muscle flap plasty. Survival after A. fumigatus mediastinitis after heart transplantation was achieved with sequential debridement in combination with topical application of antifungal agents.
\end{abstract}

Late outcome after surgical and topical treatment for Aspergillus mediastinitis after heart transplantation

Running head: Fungal mediastinitis and heart transplantation

Paul R. Vogt ${ }^{1,2}$, Raed Aser ${ }^{2}$, Daniel Schmidlin ${ }^{3}$, Paul Mohacsi ${ }^{4}$, Pascal A. Berdat ${ }^{1}$, Phillip Went ${ }^{5}$, Markus Flepp ${ }^{6}$, Carlos A. Mestres ${ }^{2}$

${ }^{1}$ Department of Cardiovascular Surgery, Klinik Im Park, Zürich, Switzerland

${ }^{2}$ Department of Cardiovascular Surgery, University Hospital Zürich, Zürich, Switzerland

${ }^{3}$ Department of Anaesthesia and Intensive Care, Klinik Im Park, Zürich, Switzerland

${ }^{4}$ Department of Cardiology, Klinik Im Park, Zürich, Switzerland

${ }^{5}$ Department of Pathology, Kantonsspital Graubünden, Chur, Switzerland

${ }^{6}$ Department of Infectious Diseases, Klinik Im Park, Zürich, Switzerland

Word Count Abstract: 102

Word Count Text: 1760

Word Count All: 2587

Address for correspondence: 


\title{
Prof. Paul R. Vogt
}

Cardiovascular Surgery

Klinik Im Park

Bellariastrasse 38

8038 Zürich (Switzerland)

Tel.: +41794009992

E-Mail: paul.vogt@hirslanden.ch

\begin{abstract}
Here we report the seven-year long-term survival after Aspergillus fumigatus mediastinitis after heart transplantation, an uncommonly described condition. A 66-year-old male developed infection withA. fumigatus covering the entire thoracic cavity with a fungal turf after orthotopic heart transplantation. Repeated surgical removal of infectious and necrotic tissue together with innovative topical treatment using voriconazole and chlorhexidine combined with systemic antifungal treatment lead to control of infection. Definitive wound closure was achieved by standard sternal refixation and latissimus dorsi muscle flap plasty. Survival after $A$. fumigatusmediastinitis after heart transplantation was achieved with sequential debridement in combination with topical application of antifungal agents.
\end{abstract}

\section{Key Words:}

Aspergillus fumigatus ; Heart transplant; Mediastinitis; Topical treatment; Fungal Infection; Mycotic aneurysm

\section{Introduction}

Despite treatment options with antifungal drugs and surgery, Aspergillus mediastinitis is generally fatal, particularly in immunocompromised patients following orthotopic heart transplantation (OHT), $[1,2]$. Herein, we describe the seven-year follow-up of a patient with early postoperative invasive Aspergillus fumigatusinfection, referred to us with sternal dehiscence after OHT. Conventional surgical debridement and intravenous antifungal treatment were thought to be hopeless due to the degree of Aspergillusdissemination, which may carry a 90-100\% mortality in immunosuppressed patients. [1,3] An innovative combination of surgical and topical antifungal treatment eventually cured the patient, allowing him to return to normal life. As this is neither a clinical trial nor an observational study, no Institutional Review Board was required. The patient gave consent for the publication of this report.

\section{Case report}

\section{Recent history and post-transplant assessment}

A 66-year-old male patient underwent $\mathrm{OHT}$ due to dilated ischemic cardiomyopathy previously treated elsewhere with multiple procedures including coronary artery bypass and left ventricular assist device (HeartWareß). OHT was performed in July 2013. Basiliximab was administered as inductive immunosuppression, followed by maintenance immunosuppression consisting of tacrolimus, mycophenolat-mofetil and prednisone. Co-morbidities included chronic gastritis, amiodarone-induced subclinical hypothyroidism, type-II diabetes mellitus, kidney cysts and chronic prostatitis, which all were well controlled. The post-transplant period was complicated by bilateral exudative pleuritis, thrombosis of right internal jugular vein, neutropenia and persistent sinus bradyarrhythmia with implantation of a two-chamber pacemaker using the left cephalic vein.

Thirty-seven days after OHT, progressive infection of the median sternotomy wound with a greyish-yellow discharge was noted starting at the epigastrium and resulting in complete skin necrosis and wound dehiscence with direct view on the transplanted heart. However, no microorganisms were identified on standard blood 
cultures. CT-scan reported pneumomediastinum and a left-sided hydropneumothorax of $300 \mathrm{ml}$ at the site of the previous LVAD, compressing the fibrotic left lower lung lobe.

The patient was referred to our clinic 43 days after OHT pre-treated with meropenem, vancomycin, linezolid, tigecycline, fluconazole and voriconazole. At clinical examination, the fully awake and neurologically normal patient was breathing spontaneously but presented in poor general condition, malnourished (serum albumin $16 \mathrm{~g} / \mathrm{L}$ ) and with renal insufficiency (creatinine $150 \mu \mathrm{mol} / \mathrm{l}$ ).

\section{Intraoperative management and postoperative course}

Surgical inspection revealed thick and pervasive, green-yellow membranes covering the entire heart, great vessels, diaphragm, both lungs as well as the entire chest wall (Fig. 1). The turf also covered contained ruptures of aorto-aortic anastomosis and the previously constructed end-to-side anastomosis of the LVAD arterial return to the native ascending aorta.

Remains of the vascular prosthesis were removed and the aorto-aortic anastomosis could be re-sutured due to sufficient length of the ascending aorta. Extensive debridement of all anatomical structures of the thoracic cavity was performed and \souta Negative Pressure Wound Therapy (NPWT) was applied.

Histopathological examination (Fig. 2), cultures as well as genomic sequencing of all specimens including pleural fluid uniformly revealed $A$. fumigatus. Galactomannan test was positive (EIA index 0.1), but bacterial fungal as well as blood cultures revealed no other microorganisms. CT-scan revealed no other organ involvement by invasive aspergillosis.

In lack of a literature report on any similar case with such extensiveAspergillus mediastinitis we empirically decided to pursue the following treatment. Surgical wound re-opening every third day, sharp and blunt mechanical scrubbing and cleaning of the fungal turf, topical application of voriconazole and chlorhexidine $2 \%, 20$-fold diluted (resulting in chlorhexidine 0.01\%), dunking the entire thoracic cavity for 40 minutes with each drug (Fig. 3) and an open chest treatment with NPWT between the re-explorations. Pre-emptive systemic antifungal therapy with a combination of caspofungin and voriconazole was started. Following susceptibility testing and determination of minimal inhibitory concentration (MIC $0.125 \mathrm{mg} / \mathrm{l}$ ), voriconazole was continued at a serum concentration of $4-6 \mathrm{mg} / \mathrm{l}$. The immunosuppressive regimen was tailored and potential organ rejection surveyed by echocardiography. Debridement and topical therapy was performed eight times. As by visible involvement of the sternal bone tissue, $5 \mathrm{~mm}$ of each sternal side was removed, histopathology confirming A. fumigatus .

After the fourth surgical session, consistent with marked visible decrease of the fungal turf overspreading the mediastinal organs, repeated microscopic and microbiological examinations were found to be sterile with regard A.fumigatus. However, colonization with multi-drug resistant Acinetobacter baumannii susceptible only to polymyxin B (MIC $0.5 \mathrm{mg} / \mathrm{l}$ ) was detected in the sixth session. Hence, a diluted colistin-solution was applied topically to the thoracic cavity and infected structures and colistin was administered intravenously for one week. The postoperative course between each surgical session was uneventful, the patient remaining isolated but fully mobilized on the regular ward.

After eight surgical revisions revealing clean intrathoracic tissues on gross examination and negative microbiological findings with respect to $A$. fumigatus, definite wound closure was achieved 26 days after the first revision with a latissimus dorsi muscle flap plasty to fill the persistent left-sided thoracic cavity. Sternal refixation and wound closure were performed according to our infection-prevention protocol (sternal wound irrigation with vancomycin wax and gentamycin solution). Careful reversal of immunosuppression allowed a stable cardiovascular course without any signs of rejection checked by repeated endomyocardial biopsy and echocardiography. The patient was discharged 47 days after our first surgical intervention with uneventful postoperative recovery and wound healing. Oral voriconazole with regular monitoring of liver function was continued for 12 months, cardiac medication and immunosuppression are continued corresponding to stateof-the-art. One year after wound closure, no findings suspicious for persistent or recurrent fungal infection on chest X-ray and CT-scan and negative serum galactomannan were reported. The patient is in excellent 
condition with good functional capacity, normal biventricular function, normal coronary angiography and no signs of cardiac rejection, seven years after this episode of mediastinitis.

\section{Discussion}

Mediastinitis is a rare but potentially lethal complication of heart transplantation, occurring in 1-10\% of cases with Staphylococcusspp. and Enterobacter spp. as the most common pathogens. [4] Our patient presented with two rare pathogens of mediastinitis, A. fumigatus and A. baumannii .

Aspergillus has been recognized as a major opportunistic pathogen and accounts for the most common mycoses in cardiac transplant recipients. [1] Diagnosis can be challenged by discriminating colonization from invasive infection and because strains morphologically identified as putative A. fumigatus are recognized as different species by molecular methods with decreased antifungal susceptibility. [5] This may lead to a delay of targeted treatment resulting in an excessive mortality. Despite negative blood cultures, invasive infection could be documented by numerous intraoperative specimens from the sternum, native and transplanted aorta including anastomosis, all intrathoracic organs and the thoracic wall. Microbiology uniformly showed characteristic culture and genomic sequencing documented hyphae consistent with Aspergillus on microscopic examination, positive serum galactomannan test and the presence of $A$. fumigatus. Hence, a diagnosis of invasive aspergillosis could be made in accordance with the definition of the US Centres for Disease Control. $[6]$

A. fumigatus mediastinitis in our patient developed within the usual time of onset of invasive aspergillosis reported to be between 36 and 52 days post transplantation. [1] Besides recent cardiac transplantation, the current patient presented with previously reported risk factors for fungal infections such as postoperative wound discharge, renal failure, administration of multiple antibiotics, ventricular assist device before surgery, as well as severe malnutrition and diabetes mellitus. $[1,3]$

Given the unique extent of the disease, the basic principle of therapy [6], i.e. intravenous antifungal treatment, tapering of immunosuppression and surgical re-exploration alone was thought not to be sufficient. With regards to the pathology of invasive aspergillosis resulting in infarction and necrosis of affected organs and poor drug delivery to tissue, our treatment regime was based on the conviction of the necessity to clean large surfaces without blood supply from thick layers of fungal material not only mechanically but also with local antiseptics.

A combination of repeated debridements with an open chest treatment, cavity baths with aqueous voriconazole and chlorhexidine, intravenous administration of voriconazole and caspofungin and lastly a chest wall reconstruction by a muscle flap plasty were necessary leading to successful recovery.

A combined systemic antifungal therapy was started with caspofungin and voriconazole, the latter guided by testing susceptibility and serum concentration. Voriconazole, a triazole with activity against yeasts and molds including Aspergillus spp. [7] performed superior when compared with amphotericin $\mathrm{B}$, and is now recommended as primary treatment of invasive aspergillosis including its uncommon manifestations such as mediastinitis and osteomyelitis. [6, 8] Voriconazole was administered systemically in the only two reported surviving cases of Aspergillus mediastinitis after OHT. [2, 4] Monitoring of its serum levels is recommended in transplanted patients because of impaired renal function and significant interactions with cyclosporine, tacrolimus and sirolimus $[1,6]$. In the current case, oral voriconazole was continued for 12 months to prevent relapse of infection, which has been reported to occur several months after seemingly successful treatment. The role of combined primary or salvage antifungal therapy is uncertain and warrants a prospective, controlled clinical trial. [6]

The use of topical voriconazole in addition to its intravenous administration has recently been found to be effective in cases of Aspergillus keratitis and endophtalmitis when administered intravitreally [9-11]. Biocidal activity of chlorhexidine against $A$. fumigatus was reported when applied topically at a concentration of $0.06 \%$ for 30 to 60 minutes and its stable and lasting adherence on human tissue without being absorbed.

[12] In addition, effectiveness of topical chlorhexidine was confirmed in the treatment of fungal keratitis 
without any adverse effects. [13] Based on this data, we decided to use chlorhexidine in the same way as the voriconazole solution as local antifungal treatment.

After the fourth surgical session, examinations revealed a sterile condition with respect to A. fumigatus . However, a superinfection with a multi-drug resistant $A$. baumannii was reported. Mediastinits due to $A$. baumannii is rare, with \soutonly two reported cases in immunocompetent subjects, successfully treated with ampicillin-sulbactam and a pan-resistant type with tigecycline. [14, 15] Notably, only one case of multi-drug resistant $A$. baumanniiin sternal wound after OHT has been reported. Favourable outcome was achieved with extensive debridement, systemic colistin and minocycline as well as wound irrigation with $5 \%$ chlorhexidine and NPWT dressing. [16] Safe and effective topical use of colistin $0.19 \%$ has been reported in the treatment of corneal infection by multi-drug resistantPseudomonas aeruginosa . [17] Hence, we used diluted colistin as described above in combination with intravenous colistin. Lastly, after the completion of topical antimicrobial therapy, a rotationallatissimus dorsi muscle flap into the thoracic cavity and complete closure of the sternum resulted in a restitutio ad integrum .

\section{Conclusion}

Our report proves that invasive $A$. fumigatus infection, yet accompanied by a superinfection with a multidrug resistant $A$. baumannii can be controlled with aggressive combined surgical, topical and systemic drug treatment even in an immunocompromised patient. The patient is alive on the long-term.

\section{Conflict of Interest Statement:}

We confirm that this manuscript is not under consideration elsewhere and none of the article content has been published previously. All authors have contributed significantly to the work, have read and approved the manuscript. All authors have given full disclosure of any conflict of interest or relationship with the industry.

\section{Data availability statement:}

The authors declare that the data collected related to this report was gathered from the institutional database and files.

\section{Source of Funding:}

The EurAsia Heart Foundation, Zürich, Switzerland, supported this work.

\section{Ethics Statement}

The patient signed a consent for publication of this report

\section{ORCID}

Carlos - A. Mestres 0000-0001-8148-9044

\section{Figure Legends}

Figure 1. Entire thoracic cavity and heart surface covered withAspergillus turf. Topical application of Chlorhexidin and Voriconazol solution into thoracic cavity.

Figure 2. Grocott and Hematoxilin \& Eosin stain showing fungus ball in the fibrinous tissue taken from the sternum, heart surface, pleura and aorta with septated hypae typical for Aspergillus fumigatus

\section{Figure 1}



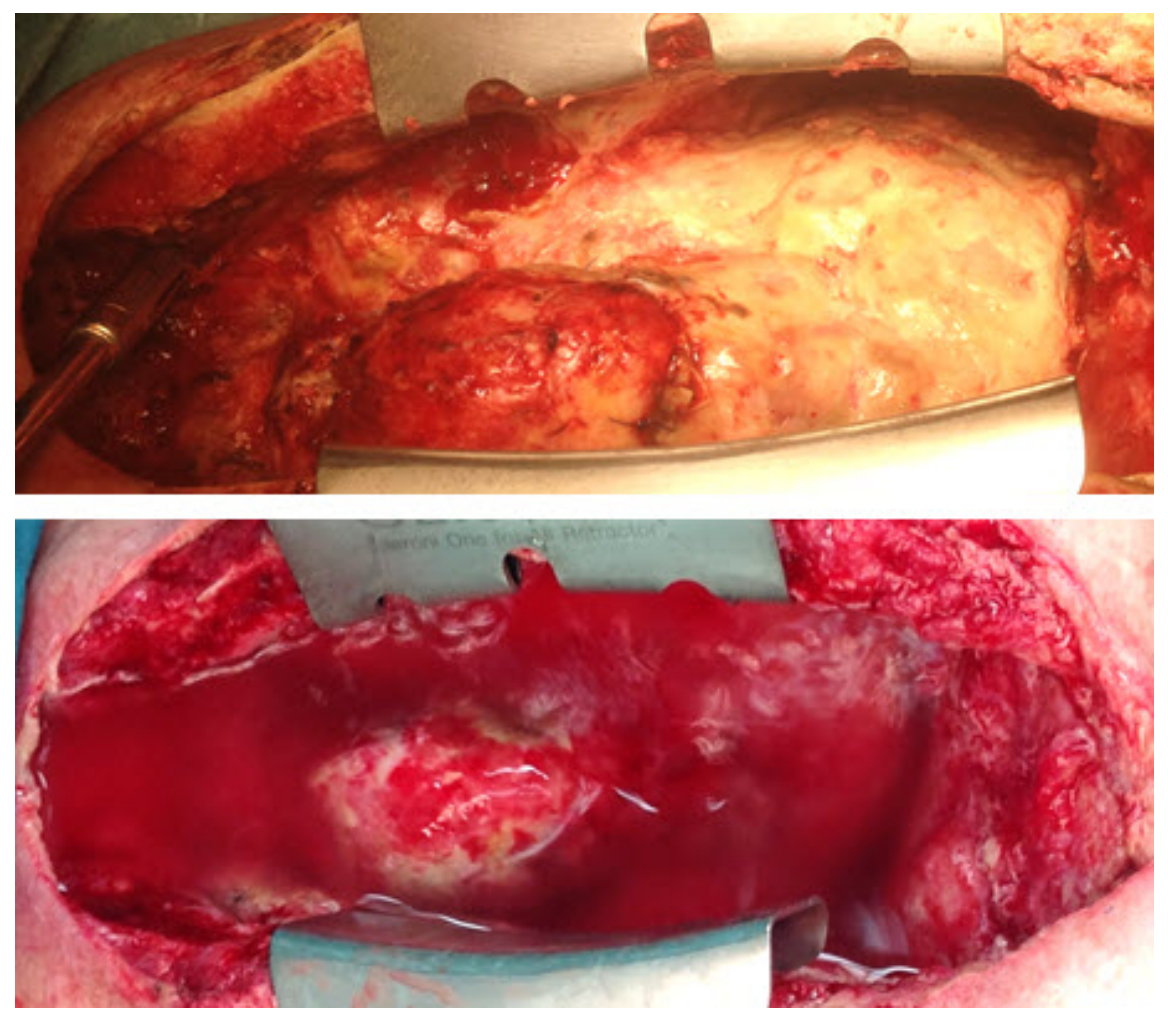

Figure 2

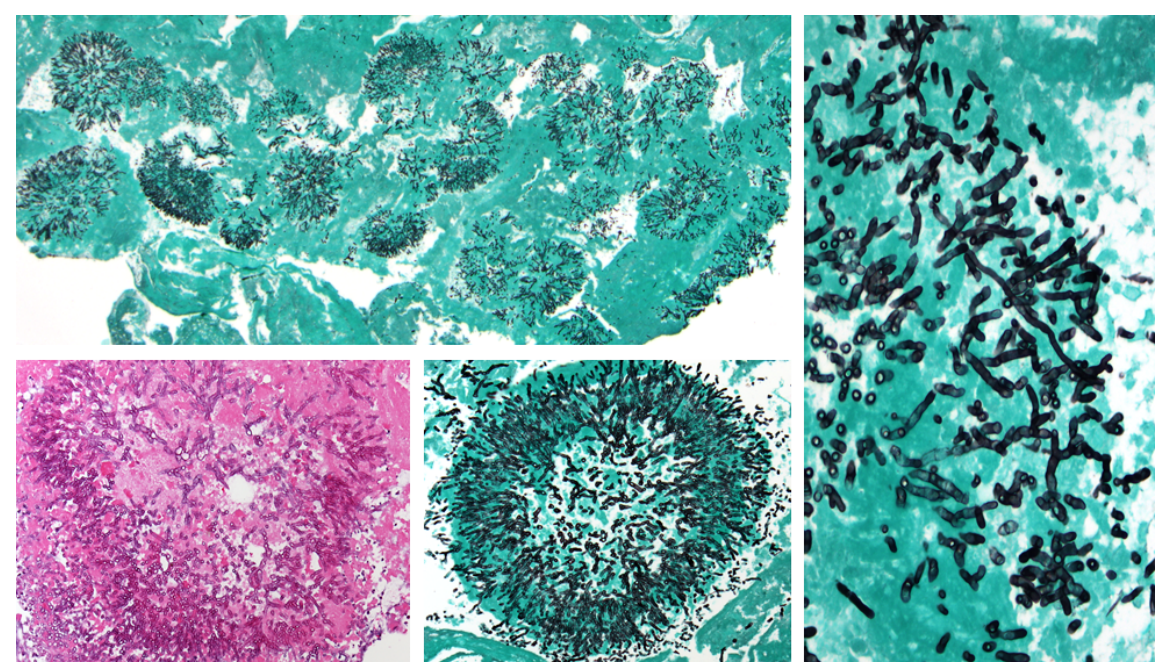

\section{References}

1. Singh N, Paterson DL. Aspergillus infections in transplant recipients. Clin Microbiol Rev 2005; 18:44-69.

2. Levin T, Suh B, Beltramo D, Samuel R. Aspergillus mediastinitis following orthotopic heart transplantation: case report and review of the literature. Transpl Infect Dis 2004; 6:129-31.

3. Chou NK, Wang JL, Chi NH, et al. Surgical treatment of mediastinitis after cardiac transplantation. 
Transplant Proc 2008; 40:2629-30.

4. Forestier E, Remy V, Lesens O, et al. A case of Aspergillus mediastinitis after heart transplantation successfully treated with liposomal amphotericin B, caspofungin and voriconazole. Eur J Clin Microbiol $2005 ; 24: 347-9$.

5. Zbinden A, Imhof A, Wilhelm MJ, et al. Fatal outcome after heart transplantation caused by Aspergillus lentulus. Transpl Infect Dis 2012; 14:E60-3.

6. Walsh TJ, Anaissie EJ, Denning DW, et al. Treatment of aspergillosis: clinical practice guidelines of the Infectious Diseases Society of America. Clin Infect Dis 2008; 46:327-60.

7. Johnson LB, Kauffman CA. Voriconazole: a new triazole antifungal agent. Clin Infect Dis 2003; 36:630-7.

8. Herbrecht R, Denning DW, Patterson TF, et al. Voriconazole versus amphotericin B for primary therapy of invasive aspergillosis. N Engl J Med 2002; 347:408-15.

9. Thiel MA, Zinkernagel AS, Burhenne J, Kaufmann C, Haefeli WE. Voriconazole concentration in human aqueous humor and plasma during topical or combined topical and systemic administration for fungal keratitis. Antimicrob Agents Chemother 2007; 51:239-44.

10. Sen P, Gopal L, Sen PR. Intravitreal voriconazole for drug-resistant fungal endophthalmitis: case series. Retina 2006; 26:935-9.

11. Jurkunas UV, Langston DP, Colby K. Use of voriconazole in the treatment of fungal keratitis. Int Ophthalmol Clin 2007; 47:47-59.

12. Tortorano AM, Viviani MA, Biraghi E, et al. In vitro testing of fungicidal activity of biocides against Aspergillus fumigatus. J Med Microbiol 2005; 54:955-7.

13. Rahman MR, Johnson GJ, Husain R, Howlader SA, Minassian DC. Randomised trial of $0.2 \%$ chlorhexidine gluconate and 2.5\% natamycin for fungal keratitis in Bangladesh. Br J Ophthalmol 1998; 82:919-25.

14. Tekce AY, Erbay A, Cabadak H, Yagci S, Karabiber N, Sen S. Pan-resistant Acinetobacter baumannii mediastinitis treated successfully with tigecycline: a case report. Surg Infect 2011; 12:141-3.

15. Lachanas E, Tomos P, Sfyras N, Miyakis S, Kostakis A. Acinetobacter baumannii mediastinitis after cardiopulmonary bypass: case report and literature review. Surg Infect 2008; 9:201-4.

16. George RS, Birks EJ, Haj-Yahia S, et al. Acinetobacter mediastinitis in a heart transplant patient. Ann Thorac Surg 2006; 82:715-6.

17. Jain R MS, Motukupally SR, Jain M. Use of topical colistin in multiple drug-resistant Pseudomonas aeruginosa bacterial keratitis. Cornea. 2014; 33:923-7. 

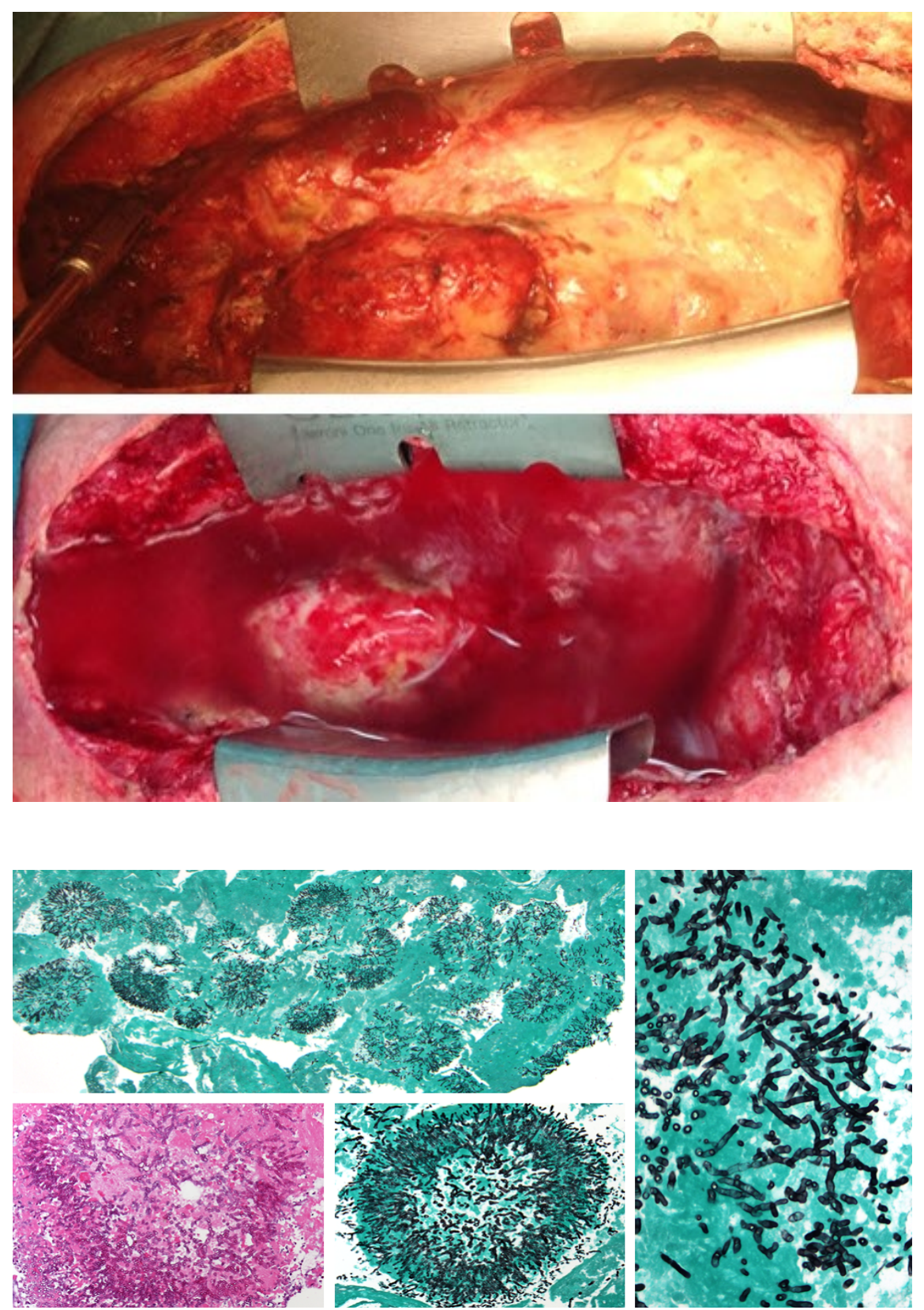